\title{
FORMATION AND DEVELOPMENT OF GLOBAL SHOCK FOR $p$-SYSTEM
}

\author{
SHUXING CHEN \\ Center of Mathematical Sciences of Zhejiang University \\ and \\ Institute of Mathematics, Fudan University \\ E-mail: sxchen@fudan.ac.cn
}

1. Introduction. Study on formation and development of shock is one of the most interesting problem in the theory of quasilinear hyperbolic systems, because it is closely related to the process of the formation of shock in physics. Mathematically, the problem is first studied in $[7,6,8]$ for single equation, then is studied in $[9,5,3]$ for system case. In these papers the process of shock and the local structure of the solution is described. However, all these discussions are restricted to the local behavior of the solution near the starting point of the shock. Obviously, the global version of such a problem is more important and inspiring. In this article we are going to study the latter. The problem to be solved is, for a time dependent quasilinear hyperbolic system, when a shock is formed from a smooth data, how does it develop and what about its asymptotic behavior. Such a problem also appears in steady supersonic flow. For instance, we studied the similar problem in paper [4] in order to analyze the stability of the weak oblique shock in steady supersonic flow. The related study on the formation of shocks can also be found in $[1,2,10]$.

We will only study Cauchy problem for $p$-system in this article. First, we will give a brief review of the result of formation and construction of shock for $p$-system in $[9,5]$ in the next section. Then we construct a series of the approximate global solutions with approximate shocks in Section 3, and prove the convergence of the series in Section 4. Finally, the asymptotic behavior of the solution is described in Section 5 .

Partially supported by NNSF, Key Grant of NMST and Doctoral Programme Foundation of Institution High Education of China.

2000 Mathematics Subject Classification: Primary 35L67, 35L65.

Key words and phrases: Formation of shocks, global existence, conservation laws, $p$-system. The paper is in final form and no version of it will be published elsewhere. 
2. Local structure of the solution. Consider the Cauchy problem of $p$-system

$$
\left\{\begin{array}{l}
\partial_{t} u-\partial_{x} v=0 \\
\partial_{t} v+\partial_{x} p(u)=0 \\
t=0: \quad u=u_{0}(x), v=v_{0}(x)
\end{array}\right.
$$

where $p(u)$ is a smooth function, satisfying $p^{\prime}<0, p^{\prime \prime}>0$. The system is strictly hyperbolic and genuinely nonlinear. Define $F(u)$ by $F^{\prime}(u)=\sqrt{-p^{\prime}(u)}$, and denote by $G(s)$ the inverse function of $F, H(s)=-p(G(s))$, then $H^{\prime}>0, H^{\prime \prime}<0$. Introduce two Riemann invariants

$$
z=\frac{1}{2}(v+F(u)), \quad w=\frac{1}{2}(v-F(u)),
$$

then (2.1) can be reduced to the following diagonalized form

$$
\left\{\begin{array}{l}
\partial_{t} z-H^{\prime}(z-w) \partial_{x} z=0 \\
\partial_{t} w+H^{\prime}(z-w) \partial_{x} w=0 \\
t=0: \quad z=z_{0}(x), w=w_{0}(x)
\end{array}\right.
$$

Like in [9] we will assume in this article that the initial data $\left(u_{0}(x), v_{0}(x)\right)$, as well as $\left(z_{0}(x), w_{0}(x)\right)$, are bounded and $C^{4}$ smooth. We assume that $w_{0}(x)$ is constant (or simply equals 0$)$, and $z_{0}(x)$ is constant outside a compact region. That is,

$$
w_{0}(x)=0, \quad z_{0}(x)=\left\{\begin{array}{l}
z_{-} \text {for } x<-M, \\
z_{+} \text {for } x>M .
\end{array}\right.
$$

Moreover, we assume that the function $g(y)=-H^{\prime}\left(z_{0}(y)\right)$ satisfies $g^{\prime}(y)>0$ for any $y$, and there exists $y^{*}$ such that

$$
g^{\prime \prime}\left(y^{*}\right)=0, \quad g^{\prime \prime \prime}\left(y^{*}\right)<0, \quad\left(y-y^{*}\right) g^{\prime \prime}(y)<0 .
$$

These conditions mean that the solution before the formation of shock is a first class simple wave, and is constant outside a compact region, moreover, the envelope of the characteristic lines of the simple wave contains only one cusp, where the shock starts (see $[9,5])$. The local construction of the solution near the cusp is given in [9], the conclusion is as follows.

Under the above assumptions there is a point $P_{0}\left(t_{0}, x_{0}\right)$, so that the problem $(2.2)$ admits a smooth solution for $t<t_{0}$, and admits a weak entropy solution for $t \geq t_{0}$, which satisfies R-H condition

$$
\sigma=-\frac{[v]}{[u]}=\frac{[p(u)]}{[v]}
$$

and Lax entropy condition on the shock front. Moreover, the shock front $x=\phi(t)$ starts from $P_{0}$, and the estimates

$$
\left\{\begin{array}{l}
\phi(t)=x_{0}+\alpha\left(t-t_{0}\right)+O\left(\left(t-t_{0}\right)^{2}\right), \\
z(t, x)=z_{0}\left(y_{0}\right)+O\left(\left(t-t_{0}\right)^{3}+\left(x-x_{0}-\alpha\left(t-t_{0}\right)\right)^{2}\right)^{1 / 6}, \\
w(t, x)=w_{0}\left(\tilde{y}_{0}\right)+O\left(\left(t-t_{0}\right)^{3}+\left(x-x_{0}-\alpha\left(t-t_{0}\right)\right)^{2}\right)^{1 / 2},
\end{array}\right.
$$

hold, where $\alpha$ is the slope of the first characteristic line through $P_{0}, y_{0}$ is the coordinate of the intersection of the first characteristics with $x$-axis. 
3. Global approximate solution. To construct the global solution to the problem (2.1) or (2.2), we start with constructing a sequence of approximate solutions, then prove the convergence of the sequence. The first approximate solution $\left(z^{(0)}(x), w^{(0)}(x)\right)$ is chosen as $w^{(0)}(x) \equiv 0$, and $z^{(0)}(x)$ is defined by the problem

$$
\left\{\begin{array}{l}
\partial_{t} z^{(0)}-H^{\prime}\left(z^{(0)}\right) \partial_{x} z^{(0)}=0 \\
t=0: \quad z^{(0)}=z_{0}(x) .
\end{array}\right.
$$

The solution should satisfy the R-H condition

$$
\sigma^{(0)}=-\left(\frac{[H(z)]}{[G(z)]}\right)^{1 / 2} .
$$

As indicated in [9], (3.1) can be written as

$$
\frac{d \phi^{(0)}}{d t}=-\left(\frac{H\left(z_{0}\left(y_{+}(t, x)\right)\right)-H\left(z_{0}\left(y_{-}(t, x)\right)\right)}{G\left(z_{0}\left(y_{+}(t, x)\right)\right)-G\left(z_{0}\left(y_{-}(t, x)\right)\right)}\right)^{1 / 2},
$$

where $x=\phi^{(0)}(t)$ is the equation of the shock, $y_{ \pm}(t, x)$ is the intersection of $x$-axis with the right (resp. left) downward characteristic line through the point $(t, x)$.

It is easy to prove that the equation (3.2) admits a global solution satisfying the initial condition $\phi\left(t_{0}\right)=x_{0}$. Moreover, since the slope of all characteristic lines is bounded and $z_{0}(x)$ is constant outside a compact interval, we can determine a number $T>0$ such that

$$
\sigma^{(0)}=-\left(\frac{H\left(z_{+}\right)-H\left(z_{-}\right)}{G\left(z_{+}\right)-G\left(z_{-}\right)}\right)^{1 / 2},
$$

for $t>T$.

The approximations $z^{(\nu+1)}$ and $w^{(\nu+1)}$ are determined by using the equation $(2.2)$, where the coefficients are substituted by $\nu$-th approximations, with suitable initial and boundary conditions. More precisely, we define

$$
\begin{gathered}
\sigma^{(\nu)}=-\left(\frac{\left[H\left(z^{(\nu)}-w^{(\nu)}\right)\right]}{\left[G\left(z^{(\nu)}-w^{(\nu)}\right)\right]}\right)^{1 / 2}, \\
\phi^{(\nu)}(t)=\int_{t_{0}}^{t} \sigma^{(\nu)}(\tau) d \tau+x_{0},
\end{gathered}
$$

then by using the coordinate transformation

$$
y=x-\phi^{(\nu)}(t)
$$

we define $z^{(\nu+1)}$ and $w^{(\nu+1)}$ by

$$
\begin{aligned}
& \left\{\begin{array}{l}
\partial_{t} z^{(\nu+1)}-\left(H^{\prime}\left(z^{(\nu)}-w^{(\nu)}\right)+\sigma^{(\nu)}\right) \partial_{y} z^{(\nu+1)}=0 \\
z^{(\nu+1)}(-1, y)=\bar{z}_{0}(y)
\end{array}\right. \\
& \left\{\begin{array}{l}
\partial_{t} w^{(\nu+1)}+\left(H^{\prime}\left(z^{(\nu)}-w^{(\nu)}\right)-\sigma^{(\nu)}\right) \partial_{y} w^{(\nu+1)}=0 \\
w^{(\nu+1)}(0, y)=\bar{w}_{0}(y) \\
w^{(\nu+1)}(t, 0)=w_{+}^{(\nu+1)}(t)
\end{array}\right.
\end{aligned}
$$

where $\bar{z}_{0}(y), \bar{w}_{0}(y)$ are the values of the solution of $(2.2)$ on $t=t_{0}, w_{+}^{(\nu+1)}$ is determined 
by $\mathrm{R}-\mathrm{H}$ condition, and can be written as

$$
w_{+}^{(\nu+1)}=m\left(z_{+}, z_{-}, w_{-}\right)[z]^{3}+w_{-}
$$

(see [9]).

As proved in $[9,5]$, there is $\epsilon>0$ such that $z^{(\nu+1)}(t, y), w^{(\nu+1)}(t, y)$ are convergent in $t_{0}+\epsilon<t<T$ with initial data given on $t=t_{0}+\epsilon$. Based on this conclusion, we can establish the following facts gradually:

(1) $z^{(\nu)}(t, y)$ are uniformly bounded,

(2) $w_{+}^{(\nu)}(t)$ are uniformly bounded,

(3) $w^{(\nu)}(t, y)$ are uniformly bounded,

(4) $\nabla z^{(\nu)}(t, y)$ and $\nabla w^{(\nu)}(t, y)$ are uniformly bounded.

4. Convergence. Let $\psi^{(\nu)}=z^{(\nu+1)}-z^{(\nu)}$, we have

$$
\left\{\begin{array}{l}
\partial_{t} \psi^{(\nu)}-\left(H^{\prime(\nu)}+\sigma^{(\nu)}\right) \partial_{y} \psi^{(\nu)}=\left(H^{\prime(\nu)}+\sigma^{(\nu)}-H^{\prime(\nu-1)}-\sigma^{(\nu-1)}\right) \partial_{y} z^{(\nu)} \\
\psi^{(\nu)}\left(t_{0}+\epsilon, y\right)=z^{(\nu+1)}\left(t_{0}+\epsilon, y\right)-z^{(\nu)}\left(t_{0}+\epsilon, y\right)
\end{array}\right.
$$

We note that the value $\alpha_{\nu}=z^{(\nu+1)}-z^{(\nu)}$ on $t=t_{0}+\epsilon$ forms a convergent series, because of the result on local existence.

Similarly, taking $\chi^{(\nu)}=w^{(\nu+1)}-w^{(\nu)}$, we have

$$
\left\{\begin{array}{l}
\partial_{t} \chi^{(\nu)}-\left(H^{\prime(\nu)}+\sigma^{\nu}\right) \partial_{y} \chi^{(\nu)}=\left(H^{\prime(\nu)}+\sigma^{(\nu)}-H^{(\nu-1)}-\sigma^{(\nu-1)}\right) \partial_{y} w^{(\nu)}, \\
\chi^{(\nu)}\left(t_{0}+\epsilon, y\right)=w^{(\nu+1)}\left(t_{0}+\epsilon, y\right)-w^{(\nu)}\left(t_{0}+\epsilon, y\right), \\
\chi^{(\nu)}(t, 0)=w_{+}^{(\nu+1)}-w_{+}^{(\nu)}
\end{array}\right.
$$

where $\beta_{\nu}=w^{(\nu+1)}\left(t_{0}+\epsilon, y\right)-w^{(\nu)}\left(t_{0}+\epsilon, y\right)$ also forms a convergent series. Moreover, in view of (3.3) we have

$$
\left|\chi^{(\nu)}(t, 0)\right| \leq C\left|\psi^{(\nu)}(t, 0)\right| .
$$

Defining $\epsilon_{\nu}=\max \left(\alpha_{\nu}, \beta_{\nu}\right)$, and

$$
a_{\nu}(t)=\max \left(\sup _{y}\left|\psi^{(\nu)}(t, y)\right|, \sup _{y}\left|\chi^{(\nu)}(t, y)\right|\right),
$$

we can establish the estimate

$$
a_{\nu}(t) \leq \int_{t_{0}+\epsilon}^{t} K a_{\nu-1}(\tau) d \tau+\epsilon_{\nu}
$$

where $K$ is a suitable constant. The inequality (4.1) implies

$$
a_{\nu}(t) \leq \epsilon_{\nu}+K \epsilon_{\nu-1} t+\ldots+K^{\nu-1} \epsilon_{1} \frac{t^{\nu-1}}{(\nu-1) !}+\frac{C K^{\nu} t^{\nu}}{\nu !} .
$$

Therefore,

$$
\sum a_{\nu}(t) \leq \sum \epsilon_{\nu} \cdot \sum \frac{K^{\nu} t^{\nu}}{\nu !}+\sum \frac{C K^{\nu} t^{\nu}}{\nu !}
$$

which leads to the convergence of the sequences $z^{(\nu)}(t, y)$ and $w^{(\nu)}(t, y)$ immediately.

Summing up the above discussion we obtain

THEOREM 4.1. For any $T>t_{0}$, there is a weak entropy solution of (2.2) with shock $x=\phi(t)$ in $t_{0}<t<T$. The solution is the extension of the local solution in $t_{0}<t<t_{0}+\epsilon$ satisfying (2.2), (2.3) and (2.4). 
5. Asymptotic behavior. Due to the arbitrariness of $T$ we obtain the global existence of the solution $z=z(t, y), w=w(t, y)$ on the whole upper half-plane. For sufficiently large $T_{1}>T$, we have

$$
\phi^{\prime}\left(T_{1}\right)=-\left(\frac{H\left(z_{+}\right)-H\left(z_{-}\right)}{G\left(z_{+}\right)-G\left(z_{-}\right)}\right)^{1 / 2},
$$

and

$$
z\left(T_{1}, x\right)=\left\{\begin{array}{lll}
z_{+} & \text {for } & x>\phi(t), \\
z_{-} & \text {for } & x<\phi(t) .
\end{array}\right.
$$

Therefore, when $t \geq T_{1}$, the solution is constant in the domain left to the shock, and is a second class simple wave in the domain right to the shock.

On the other hand, the solution of the Riemann problem of the system (2.2) with initial value

$$
(z, w)= \begin{cases}\left(z_{-}, 0\right) & \text { for } \quad x<0 \\ \left(z_{+}, 0\right) & \text { for } \quad x<0\end{cases}
$$

is composed of a left shock and a right simple wave. Denote the solution by $(Z, W)$, which is a self-similar solution, and is composed of a first class shock and a second class center wave. Then we confirm that the solution $(z, w)$ asymptotically approaches $(Z, W)$ in the following sense: for any compact set $\Omega$ in $t>0$, we have

$$
\lim _{\alpha \rightarrow \infty}\left|\left(Z\left(\frac{x}{t}\right), W\left(\frac{x}{t}\right)\right)-(z(\alpha t, \alpha x), w(\alpha t, \alpha x))\right| \rightarrow 0
$$

for $(t, x) \in \Omega$. This is the right description of the asymptotic behavior of the solution $(z, w)$.

\section{References}

[1] S. Alinhac, Blowup for Nonlinear Hyperbolic Equations, Progr. Nonlinear Differential Equations Appl. 17, Birkhäuser, Boston-Basel-Berlin, 1995.

[2] S. Alinhac, Mécanisme d'explosion des solutions classiques de systèmes hyperboliques unidimensionnels, Séminaire sur les Équations aux Dérivées Partielles, 1994-95, Exp. No. VI, École Polytech., Palaiseau, 1995.

[3] S. X. Chen, How does a shock in supersonic flow grow out of smooth data?, J. Math. Phys. 42 (2001), 1154-1172.

[4] S. X. Chen, Stability of weak oblique shock front, Sci. China Ser. A 45 (2002), 1012-1019.

[5] S. X. Chen, L. M. Dong, Formation and construction of shock for p-system, Sci. China Ser. A 44 (2001), 1139-1147.

[6] S. X. Chen, Z. B. Zhang, On the generation of shock waves of first order quasilinear equation, J. Fudan Natur. Sci. (1963), 13-22.

[7] R. Courant, K. O. Friedrichs, Supersonic Flow and Shock Waves, Interscience, New York, 1948.

[8] P. D. LAx, Development of singularities of solutions of nonlinear hyperbolic partial differential equations, J. Math. Phys. 5 (1964), 611-613.

[9] M.-P. Lebaud, Description de la formation d'un choc dans le p-système, J. Math. Pures Appl. (9) 73 (1994), 523-565.

[10] S. NAKAne, Formation of shocks for a single conservation law, SIAM J. Math. Anal. 19 (1988), 1391-1408. 